\title{
Effect of Chronic Tobacco Smoking on Hearing among Men
}

\author{
*Sanjay Kumar Munjal, Parul Sud, Anuradha Sharma and Naresh K Panda \\ Department of Otolaryngology, Post Graduate Institute of Medical Education and Research, India
}

Submission: April 29, 2017; Published: May 11, 2017

*Corresponding author: Sanjay Kumar Munjal, Associate Professor and Incharge, Speech and Hearing Unit, Department of Otolaryngology, Post Graduate Institute of Medical Education and Research, Chandigarh, India, Tel: +91-9815653262; +91-172-2756955; Fax: +91-172-2744401; 2744450; Email: sanjaymunjal1@hotmail.com

Abstract

Cigarette smoking is a well known health problem affecting various body organs. There is a dearth of literature on early changes in auditory functioning at high frequency due to smoking. Hence, the present study aimed to investigate the audiological parameters for 100 chronic smokers. Their results were compared to 50 non smokers. Audiological test battery included conventional pure tone audiometry, extended high frequency audiometry (up to $16 \mathrm{KHz}$ ), Distortion Product Otoacoustic Emission (DPOAE) and Transient Evoked Otoacoustic Emission (TEOAE). Results revealed a significant difference between the two groups on conventional and extended high frequency pure tone audiometry. Statistically significant differences were observed between the two groups for DPOAEs and TEOAEs. The results revealed that chronic smokers have impaired hearing at high as well as low frequencies. Smoking may also lead to cochlear dysfunction as revealed by significant difference in OAEs responses between smokers and non-smokers.

Keywords: Chronic smoking; Tobacco smoking; Sensori neural hearing loss; Impedance audiometry; Oto acoustic emissions; High frequency audiometry

Abbreviations: DPOAE: Distortion Product Otoacoustic Emission; TEOAE: Transient Evoked Otoacoustic Emission; WHO: World Health Organization; HFA: High Frequency Audiometry; OAE’s: Otoacoustic Emissions; SN: Sensorineural, CHL: Conductive Hearing Loss; MHL: Mixed Hearing Loss; DPOAE: Distortion Product Otoacoustic Emission; TEOAE: Transient Evoked Otoacoustic Emission; IHS: Intelligent Hearing System; SNR: Signal-to-Noise Ratio; CDC: Center for Disease Control and Prevention; PTA: Puretone Audiometry

\section{Introduction}

Tobacco smoking is one of the biggest public health threats that the world is facing at present. The estimated prevalence of current tobacco smoking in India, among persons aged 15 years and older is $20.4 \%$, (World Health organization, 2015). The hazardous effects of tobacco smoking are well known across the world. The World Health organization (WHO) reports 6 million deaths per year due to tobacco usage (more than 5 million from direct tobacco usage and more than 600000 as non smokers, exposed to second hand smoke) [1]. The tobacco smoking epidemic is on a rise. The effects of cigarette smoking are destructive and widespread. Its detrimental effects in the form of disease related to chronic tobacco intake have just begun. Smoking of cigarettes harms nearly every organ of the body causing many diseases and reduces the general health of smokers [2]. The number of deaths reported because of tobacco usage is far more than from HIV, illegal drug and alcohol use, motor vehicle injuries, suicides and murders collectively.
Chronic smokers usually die during their middle age due to its deleterious effects on several organs [3]. Many of these health effects are related to exposure to drug, its usage, nicotine content, number of cigarette intake per day. There are approximately 4000 chemicals in cigarette smoke and many of them are toxic [2]. These include nicotine, formaldehyde, benzene, arsenic, vinyl chloride, ammonia and hydrogen cyanide via second hand smoke inhalation (CDC, 2006). Nicotine reaches the brain within 10 seconds following inhalation, and travels to every part of the body, even in breast milk [2].

Intake of nicotine is reported to have direct ototoxic effect. It leads to cochlear ischemia with lower blood oxygen levels, vascular obstructions, and altered blood viscosity [4]. This vascular insufficiency in the cochlear organ may lead to progressive sensori neural hearing loss [2]. Reduction in oxygen supply to the organ of corti leads to reduced energy available for the cochlea and possibly leads to more hair cell injury [4-6]. 
Smokers are at a $70 \%$ greater risk of developing hearing loss than non smokers. Mc Reynold [7-9]. The health risk behavior survey conducted by the Center for Disease Control and Prevention (CDC, $2007 ; 2008$ ) indicates that approximately $10 \%$ of students in grades 9 through 12 currently smoke more than 10 cigarettes per day. In the 18-64 year age range the prevalence increases to approximately $20 \%$, with a slightly lower prevalence rate in females than in males across the age span (CDC, 2007; 2008). Moreover, estimates of women who smoke during pregnancy indicate an overall prevalence of $11.4 \%$, with a range of $6.3 \%$ to as high as $26.2 \%$ when state-specific data are evaluated (CDC, 2004). Cruickshanks et al. [10], 2003 conducted a longitudinal study on cigarette smokers. Results revealed that cigarette smokers were 1.69 times more likely to have hearing loss than nonsmokers. It has also been reported that second hand smoke exposure was associated with elevated puretone hearing thresholds for low/mid and high frequencies Fabry et al. [11].

Routinely, several tests are used to assess the peripheral auditory system. Conventional pure tone threshold audiometry allows the investigation of pure tones at 250 to $8000 \mathrm{~Hz}$. High frequency audiometry (HFA) measures hearing above $8000 \mathrm{~Hz}$ and assists in the assessment of cochlear response at the basal end. The high frequency audiometry is a valuable clinical tool in assessing early auditory involvement $[12,13]$. Otoacoustic Emissions (OAE's) provide direct insight into outer hair cell cochlear amplification; and normal cochlear biologic mechanism activity [14]. OAEs also assist in detecting early cochlear hair cell changes. Hearing loss related to smoking and passive smoking has been reported earlier. Sharabi et al. [15], 2001 in their study, reported conductive hearing loss in all subject groups (20-68 years). Overall, $6.1 \%$ of current and past smokers experienced conductive hearing loss of at least a mild degree ( $>30 \mathrm{~dB}$ across the $0.25-8 \mathrm{kHz}$ range). The authors also reported that smokers were twice more likely to experience a mild sensorineural hearing loss ( $>25 \mathrm{~dB}$ either in the low or high frequencies) compared to nonsmokers [15].

Nakanishi, Okamoto, Nakamura, Suzuki, and Tatara [16] showed that male office workers in the 30-59 year age range who smoked were at a greater risk for hearing loss at $4 \mathrm{kHz}$ than at $1 \mathrm{kHz}$ compared to nonsmokers and ex-smokers. In their study, they controlled for age and a number of health factors like body mass index, alcohol consumption, triglyceride levels, etc., and showed that as numbers of cigarettes smoked per day and pack years of smoking increased, the risk for high-frequency hearing loss increased in a dose dependent manner, whereas lowfrequency hearing loss remained unchanged. Negley, Katbamna, Crumpton, and Lawson, [17] likewise, showed no differences in ultra high-frequency hearing of smokers and nonsmokers in the 20-30years age range. Smoking related hearing loss in frequencies higher than $8 \mathrm{kHz}$ is less studied, especially in Indian population. There is a dearth of data reporting HFA and OAE finding in smokers and correlating the same with other audiological tests. Hence the present study was conducted to detect early hearing changes in smokers.

\section{Materials and Method}

The present study was carried out in Speech and Hearing Unit, Department of Otorhinolaryngology, Postgraduate Institute of Medical Education and Research, Chandigarh (India). The sample was divided into two groups: the study group and the control group. The study group included 100 chronic smokers with more than 10 cigarettes per day for a period of more than 5 years. The age range was from 24 years to 40 years with a mean age of 31.2 years. The control group consisted of 50 normal hearing individuals with no history of cigarette smoking. The age range was from 20 years to 31 years with a mean age of 24.5 years. Both the groups included only males. Subjects with any middle ear/inner ear pathology, ototoxicity, noise exposure, diabetes mellitus, renal failure, cardiovascular diseases, and any other conditions that can lead to hearing loss other than smoking were excluded from the study. Informed consent was obtained from all the subjects and the study was approved by the ethical board of the institute.

All participants underwent a comprehensive otological examination to rule out the presence of any otological problems leading to hearing loss. The audiological test battery included conventional puretone audiometry with high frequency audiometry (HFA), tympanometry and otoacoustic emission (OAE) evaluations. All the audiological tests were carried out in sound treated rooms. Puretone audiometry (PTA) test was conducted with a diagnostic audiometer Madsen Orbiter 922(Denmark), for test frequencies from $250 \mathrm{~Hz}$ to 8,000 Hz. PTA was carried out using TDH 39, (Telephonics) supra-aural earphones with noise excluding headsets. A hearing threshold of $<25 \mathrm{dBHL}$ was considered as normal hearing (World Health Organization, Grades of hearing impairment, 2008) [18]. The puretone average was calculated for $500 \mathrm{~Hz}, 1,000 \mathrm{~Hz}$ and $2,000 \mathrm{~Hz}$. Extended high frequency (HFA) was performed for the test frequencies of $10 \mathrm{KHz}, 12 \mathrm{KHz}, 14 \mathrm{KHz}$ and $16 \mathrm{KHz}$, using HDA200, (Sennheiser) earphones. Bone conduction hearing thresholds were estimated using bone vibrator B71, (Radio Ear).

The air bone gap obtained with air conduction and bone conduction thresholds confirmed the type of hearing loss as Sensorineural (SN), conductive hearing loss (CHL) and mixed hearing loss (MHL). Tympanometry test was conducted with Madsen Zodiac 901 tympanometer calibrated according to ANSI 1992 standards. A low frequency probe tone $(226 \mathrm{~Hz})$ with pressure variations from -400 to 200 dapa was used for testing. Tympanogram obtained were classified according to Jerger and Jerger classification system [Jerger, 1970]. Tympanometric evaluation was done to rule out the presence of any underlying conductive pathology in the outer and middle ear.

OAE was also a part of the audiological test battery to test the integrity of the outer hair cells. Distortion Product Otoacoustic 
Emission (DPOAE) and Transient Evoked Otoacoustic Emission (TEOAE) were performed with this aim.OAE was measured in a sound treated room using the Intelligent Hearing System (IHS), USA. For DPOAE testing the signal-to-noise ratio (SNR) and amplitude of DPOAE were studied. Frequencies analyzed were $0.5 \mathrm{KHz}, 0.7 \mathrm{KHz}, 1 \mathrm{KHz}, 1.4 \mathrm{KHz}, 2 \mathrm{KHz}, 3 \mathrm{KHz}, 4 \mathrm{KHz}, 6 \mathrm{KHz}$ and $8 \mathrm{KHz}$. An SNR value of more than 3 at three consecutive frequencies was taken as present DPOAE. TEOAE were analyzed using click stimulus. The frequencies analyzed were $1 \mathrm{KHz}, 1.5$ $\mathrm{KHz}, 2 \mathrm{KHz}, 3 \mathrm{KHz}$ and $4 \mathrm{KHz}$. An SNR value of more than 3 in three consecutive frequencies was taken as present TEOAE. A statistical analysis using unpaired' $t$ ' test was applied to evaluate the difference between the study group and the control group for conventional puretone audiometry, extended high frequency audiometry, DPOAE and TEOAE.

\section{Results}

\section{Puretone Audiometry}

All participants in the present study (study and control group) had puretone average of less than $25 \mathrm{~dB}$ HL on conventional puretone audiometry. Unpaired ' $t$ ' test was used to investigate the statistical significant difference between the two groups. (Table 1 presents a comparison between the study group and the control group for Conventional puretone audiometry. The results reveal a statistically significant difference between the study and the control group for the right ear at $0.25 \mathrm{KHz}, 0.5$ $\mathrm{KHz}, 2 \mathrm{KHz}$ and $4 \mathrm{KHz}$. However, no significant difference was observed at $1 \mathrm{KHz}$ and $8 \mathrm{KHz}$. A statistically significant difference was observed between the two groups for all frequencies $(0.25$ $\mathrm{KHz}, 0.5 \mathrm{KHz}, 1 \mathrm{KHz}, 2 \mathrm{KHz}, 4 \mathrm{KHz}$ and $8 \mathrm{KHz}$ ) for the left ear extended High Frequency Audiometry.

Table 1: Comparison of study group and control group for Conventional Pure Tone Audiometry.

\begin{tabular}{|c|c|c|c|c|c|c|}
\hline \multirow[b]{2}{*}{ Frequency (khz) } & \multirow[t]{2}{*}{ EAR } & \multicolumn{2}{|c|}{ Study Group $(n=100)$} & \multicolumn{2}{|c|}{ Control Group $(n=50)$} & \multirow{2}{*}{ 't' VALUE } \\
\hline & & $\operatorname{Mean}(\mathrm{dB})$ & S.d & $\operatorname{Mean}(\mathrm{dB})$ & S.d & \\
\hline \multirow{2}{*}{0.25} & Right & 12.71 & 5.51 & 9.50 & 4.65 & $2.11^{*}$ \\
\hline & Left & 15.63 & 5.17 & 9.25 & 3.35 & $4.92^{* * *}$ \\
\hline \multirow{2}{*}{0.5} & Right & 12.29 & 5.51 & 8.75 & 3.19 & $2.66^{*}$ \\
\hline & Left & 14.38 & 5.38 & 9.75 & 4.44 & $3.12^{* *}$ \\
\hline \multirow{2}{*}{1} & Right & 11.88 & 4.62 & 9.75 & 3.80 & 1.68 \\
\hline & Left & 12.08 & 4.40 & 9.50 & 3.59 & $2.14^{*}$ \\
\hline \multirow{2}{*}{2} & Right & 13.54 & 5.00 & 9.75 & 4.13 & $2.76^{* *}$ \\
\hline & Left & 14.38 & 5.38 & 9.00 & 3.48 & $3.20^{* *}$ \\
\hline \multirow{2}{*}{4} & Right & 16.46 & 5.80 & 11.50 & 4.01 & $3.33^{* *}$ \\
\hline & Left & 16.04 & 4.89 & 7.75 & 3.43 & $6.60^{* * *}$ \\
\hline \multirow{2}{*}{8} & Right & 17.50 & 5.11 & 13.00 & 9.51 & 1.90 \\
\hline & Left & 17.50 & 6.92 & 9.00 & 3.84 & $5.15^{* * *}$ \\
\hline
\end{tabular}

${ }^{*} \mathrm{p}<0.05,{ }^{* *} \mathrm{p}<0.01,{ }^{* * *} \mathrm{p}<0.001$

KHz: Kilo Hertz; dB: Decibel

Extended High Frequency Audiometry was carried out to investigate the effect of cigarette smoking on the extended high frequencies. $41 \%$ of the subjects in the study group had mild degree of hearing loss (26-40 dB) in right ear; moderate degree of hearing loss (41-55 dB) in 8 subjects $(8 \%)$ and moderate to severe degree of hearing loss (56-70 dB) in 16(16\%) subjects The results of the left ear for the study group revealed a mild degree of hearing loss (25-40 dB) in 66 (66\%) and moderate (41-
$55 \mathrm{~dB})$ in $4(4 \%)$ of the subjects respectively. The control group had no significant hearing loss in both ears for the extended high frequencies. An unpaired ' $t$ ' test was used to investigate the difference between the study and control group for extended high frequencies. A statistically significant difference $(p<0.001)$ was observed between the two groups for all the tested extended high frequencies i.e. $10 \mathrm{KHz}, 12 \mathrm{KHz}, 14 \mathrm{KHz}$ and $16 \mathrm{KHz}$ in both the right ear and the left ear (Table 2).

Table 2: Comparison of study group and control group for Extended High Frequency Audiometry.

\begin{tabular}{|c|c|c|c|c|c|c|}
\hline \multirow{2}{*}{ Frequency (khz) } & \multirow{2}{*}{ Ear } & \multicolumn{2}{|c|}{ Study group(n=100) } & \multicolumn{2}{|c|}{ Control group(n=50) } & \multicolumn{2}{c|}{ 't' value } \\
\cline { 3 - 8 } & & Mean(db) & S.d & Mean(db) & 7.34 & $7.05^{* * *}$ \\
\hline \multirow{2}{*}{10} & Right & 23.33 & 7.76 & 7.25 & 3.80 & $7.75^{* * *}$ \\
\cline { 2 - 8 } & Left & 24.38 & 9.81 & 7.50 & 6.96 & $7.64^{* * *}$ \\
\hline \multirow{2}{*}{12} & Right & 27.29 & 10.53 & 7.00 & 6.86 & $5.32^{* * *}$ \\
\cline { 2 - 7 } & Left & 23.25 & 11.03 & 8.75 & & \\
\hline
\end{tabular}




\section{Global Journal of Otolaryngology}

\begin{tabular}{|c|c|c|c|c|c|c|}
\hline \multirow{2}{*}{14} & Right & 37.29 & 17.69 & 9.00 & 7.71 & $7.08^{* * *}$ \\
\cline { 2 - 7 } & Left & 33.13 & 14.58 & 7.50 & 8.81 & $7.18^{* * *}$ \\
\hline \multirow{2}{*}{16} & Right & 40.00 & 15.97 & 8.25 & 8.47 & $8.01^{* * *}$ \\
\cline { 2 - 7 } & Left & 40.79 & 18.35 & 7.50 & 9.53 & $7.06^{* * *}$ \\
\hline
\end{tabular}

${ }^{*} \mathrm{p}<0.05,{ }^{* *} \mathrm{p}<0.01,{ }^{* * *} \mathrm{p}<0.001$

KHz: Kilo Hertz; dB: Decibel

\section{Otoacoustic Emissions}

The cochlear integrity was checked using the OAEs. DPOAE and TEOAE were performed with a similar aim for all the participants. (Table 3) shows a comparison between study group and control group for SNR values of DPOAEs. A statistically significant difference $(\mathrm{p}<0.01)$ was obtained between the two groups at $2 \mathrm{KHz}$ and $4 \mathrm{KHz}$ for the right ear. A statistically significant difference $(p<0.01)$ was obtained between the two groups at frequencies $0.7 \mathrm{KHz}, 1 \mathrm{KHz}$ and $6 \mathrm{KHz}$. No significant difference was observed at $0.5 \mathrm{KHz}, 1.4 \mathrm{KHz}, 2 \mathrm{KHz}, 3 \mathrm{KHz}, 4 \mathrm{KHz}$, and $8 \mathrm{KHz}$.

Table 3: Comparison of study group and control group for SNR values of DPOAE.

\begin{tabular}{|c|c|c|c|c|c|c|}
\hline \multirow[b]{2}{*}{ Frequency (khz) } & \multirow[b]{2}{*}{ Ear } & \multicolumn{2}{|c|}{ Study group $(n=100)$} & \multicolumn{2}{|c|}{ Control group $(n=50)$} & \multirow{2}{*}{ 't' VALUE } \\
\hline & & Mean(dbspl) & Sd & Mean(dbspl) & Sd & \\
\hline \multirow{2}{*}{0.5} & Right & 1.46 & 9.07 & 2.00 & 8.62 & 0.20 \\
\hline & Left & 0.50 & 4.18 & 2.85 & 7.41 & 1.26 \\
\hline \multirow{2}{*}{0.7} & Right & 4.63 & 5.82 & 8.40 & 9.21 & 1.59 \\
\hline & Left & 4.33 & 6.61 & 9.45 & 8.02 & $2.28^{* *}$ \\
\hline \multirow{2}{*}{1} & Right & 4.38 & 8.69 & 9.40 & 11.13 & 1.64 \\
\hline & Left & 3.96 & 9.91 & 11.20 & 8.24 & $2.65^{* *}$ \\
\hline \multirow{2}{*}{1.4} & Right & 10.42 & 9.09 & 15.45 & 7.67 & 1.2 \\
\hline & Left & 11.92 & 10.09 & 17.30 & 8.85 & 1.89 \\
\hline \multirow{2}{*}{2} & Right & 8.25 & 7.31 & 15.35 & 8.17 & $3.00^{* *}$ \\
\hline & Left & 11.79 & 9.39 & 15.25 & 7.69 & 1.34 \\
\hline \multirow{2}{*}{3} & Right & 13.88 & 9.06 & 18.60 & 7.29 & 1.91 \\
\hline & Left & 12.83 & 9.41 & 17.50 & 9.38 & 1.64 \\
\hline \multirow{2}{*}{4} & Right & 8.25 & 7.40 & 17.90 & 10.20 & $3.53^{* *}$ \\
\hline & Left & 9.50 & 6.64 & 14.55 & 7.30 & $2.38^{* *}$ \\
\hline \multirow{2}{*}{6} & Right & 8.63 & 7.01 & 11.60 & 7.26 & 1.38 \\
\hline & Left & 7.63 & 5.48 & 7.65 & 6.98 & 0.01 \\
\hline \multirow{2}{*}{8} & Right & 5.58 & 7.65 & 6.20 & 5.42 & 0.31 \\
\hline & Left & 4.79 & 4.44 & 3.15 & 6.83 & 0.92 \\
\hline
\end{tabular}

${ }^{*} \mathrm{p}<0.05,{ }^{* *} \mathrm{p}<0.01,{ }^{* * *} \mathrm{p}<0.001$

KHz: Kilo Hertz; dB: decibel; SNR: Signal- to- noise ratio; DPOAE: Distortion Product Otoacoustic Emissions

(Table 4) shows a comparison between the two groups for SNR values of TEOAE for both ears. The results reveal a

Table 4: Comparison of study group and control group for SNR values of TEOAEs.

\begin{tabular}{|c|c|c|c|c|c|c|}
\hline \multirow{2}{*}{ Frequency (khz) } & \multirow{2}{*}{ Ear } & \multicolumn{2}{|c|}{ Study group $(n=100)$} & \multicolumn{2}{|c|}{ Control group $(n=50)$} & \multirow{2}{*}{ 't' value } \\
\hline & & Mean(dbspl) & S.d & Mean(dbspl) & S.d & \\
\hline \multirow{2}{*}{1} & Right & 0.69 & 2.91 & 2.07 & 3.35 & 1.44 \\
\hline & Left & 1.74 & 3.88 & 3.89 & 3.08 & $2.44^{*}$ \\
\hline \multirow{2}{*}{1.5} & Right & 0.90 & 4.24 & 5.05 & 3.50 & $3.56^{* *}$ \\
\hline & Left & 4.10 & 4.38 & 5.47 & 4.18 & 1.06 \\
\hline \multirow{2}{*}{2} & Right & 2.97 & 4.06 & 7.12 & 4.11 & $3.36^{* *}$ \\
\hline & Left & 2.67 & 4.11 & 7.05 & 4.75 & $3.23^{* *}$ \\
\hline
\end{tabular}

statistically significant difference in the right ear between the two groups at frequency $2 \mathrm{KHz}$ and $3 \mathrm{KHz}$. Comparison of SNR values of TEOAE in the left ear showed a significant difference between the means of the study group and the control group at $1 \mathrm{KHz}, 2 \mathrm{KHz}, 3 \mathrm{KHz}$, and $4 \mathrm{KHz}$. No significant difference was und between the two groups at $1.5 \mathrm{KHz}$. 


\section{Global Journal of Otolaryngology}

\begin{tabular}{|c|c|c|c|c|c|c|}
\hline \multirow{3}{*}{3} & Right & 0.46 & 3.21 & 6.82 & 3.66 & $6.07 * * *$ \\
\cline { 2 - 8 } & Left & 4.15 & 3.23 & 8.98 & 4.34 & $4.10^{* * *}$ \\
\hline \multirow{2}{*}{4} & Right & 0.70 & 1.90 & 1.80 & 2.08 & 1.81 \\
\cline { 2 - 8 } & Left & 1.01 & 1.95 & 2.86 & 3.28 & $2.20^{*}$ \\
\hline
\end{tabular}

${ }^{*} \mathrm{p}<0.05,{ }^{* *} \mathrm{p}<0.01,{ }^{* * *} \mathrm{p}<0.001$

KHz: Kilo Hertz; dB: Decibel; SNR: Signal- to- noise ratio; TEOAE: Transient Evoked Otoacoustic Emissions

\section{Tympanometry}

Tymapnometry test was done for all participants in the study to rule out any middle ear pathology. All the subjects had a type A tympanogram suggestive of normal middle functioning. The unpaired ' $\mathrm{t}$ ' test did not show any statistical significant difference between the two groups for the various tympanometric parameters viz. compliance, middle ear pressure and ear canal volume.

\section{Discussion}

The present study was carried out with an aim to investigate the effect of chronic smoking on the hearing mechanism of adult males in an Indian population. Although various studies have been conducted to predict the hearing status among chronic smokers $[4,9,14,15]$ and second hand smokers [11], but inconsistent findings among different research studies necessitated this study for an Indian population. Kumar A, Gulati $\mathrm{R}$, Singhal S. et al. [19-23] reported sensori neural hearing loss in $77.5 \%$ subjects, mixed in $18.3 \%$ subjects. Gaur et al. [24] 2012 also reported greater risks for middle ear and inner ear disorders for tobacco users. De Oliveira et al., 2009 also revealed that although within normal ranges, auditory thresholds in low frequencies were higher among smokers. They also noticed a marked increase in thresholds at higher frequencies [19]. The results of the present study reveal high frequency hearing loss for the chronic smokers group when compared to the control group.

Although the hearing thresholds were within normal limits for the both groups, a statistically significant difference was observed between the two groups. Mizoue et al. [20] 2003 reported that a dose response relation at $4000 \mathrm{KHz}$ indicated that smoking was significantly associated with an increased risk of high frequency loss. They also explained that smoking damages hair cells through an ischaemic mechanism that is by reducing blood flow to the cochlea by increasing carboxyhaemoglobin. Histopathological studies have shown changes within the nuclei of the cells of the Scarpa's ganglion, peripheral nerves and the terminal nerve apparatus [21]. Tobacco smoke causes obliteration of end arteries at the level of otic end organ resulting in progressive sensorineural hearing loss [22] It is hypothesized that the poorer response in the low frequencies may be due to the ischemic mechanism or the ototoxic effects of nicotine on hair cell function not only on the basal end of the cochlea but with the increase in duration of smoking, damage spreads to the apical end of the cochlea. Nakanishi et al., 2000 also conducted a 5 year follow up of Japanese cigarette smoking male office workers and indicated that smoking is highly associated with development of high frequency hearing impairment [16]. Cruikshanks et al. [10], 2003 also suggested that in older adults, cigarette smokers were 1.69 times more likely to have hearing loss as compared to nonsmokers.

In this present study, a statistically significant difference was obtained between the two groups for frequencies $0.25 \mathrm{KHz}, 0.5$ $\mathrm{KHz}, 1 \mathrm{KHz}, 2 \mathrm{KHz}, 4 \mathrm{KHz}, 8 \mathrm{KHz}, 10 \mathrm{KHz}, 12 \mathrm{KHz}, 14 \mathrm{KHz}$, and 16 $\mathrm{KHz}$. Even though the study group and the control group revealed a normal hearing, there was a significant difference between the two groups for Conventional PTA, whereas, on HFA most of the smokers showed a hearing loss. Similar results were found by Paschoal et al. [14] (2009) that on (High Frequency Audiometry) HFA smokers had worse thresholds for all frequencies, and statistically significant differences at $12,500 \mathrm{~Hz}$ on right ears and $14000 \mathrm{~Hz}$ on both ears. Ohgami et al. [25], 2011 reported that even light smoking might affect hearing level at $12 \mathrm{kHz}$. The cochlear physiology shows that higher the frequency of sound stimulation, the closer is the stimulation to the basal region of the cochlea. It is thus believed that conventional audiometry (250-8 KHz) assesses predominantly the medial portion of the cochlea, while HFA looks into basal portion of the cochlea [26]. It reveals that in smokers the cochlear damage begins at basal end. Therefore, HFA should be indispensable part of audiological test battery.

The cochlear status with respect to cigarette smoking was assessed using OAE. DPOAE and TEOAE were used for this purpose. The study revealed decreased SNR values for DPOAE and TEOAE as well as decreased amplitude of DPOAE responses. Decreased amplitude and SNR values depict cochlear changes especially at outer hair cells. Reduction in otoacoustic emission responses could be due to lack of oxygen in the blood of the hair cells, which cause hair cells to breakdown and result in hearing loss [17]. Mehrparvar AH et al. [27] 2015 have also reported mean DP-OAE amplitude significantly higher in smokers for frequencies above $1000 \mathrm{~Hz}$, in comparison to non smokers. Their study concluded that smoking can aggravate the effect of noise on hearing. Paschoal et al. [14] 2009 also studied effect of cigarette smoking on OAEs. They reported that smokers presented a lower response level in the frequencies of $1 \mathrm{kHz}$ in both ears and $4 \mathrm{kHz}$ in the left ear. The results further revealed that cigarette smoking has an adverse effect on cochlear function and leads to tinnitus in certain cases. Aha et al. found positive staining with 8-Oxo G and hypoxia inducible factor 100 in the inner ear of the smoking only and smoking- plus- noise group similar to noise 
only-mice. They concluded that smoking disturbs the recovery mechanism in the cochlea [24].

The epidemic caused by smoking has already started taking its course with current smokers being at $15.1 \%$ greater risk of developing hearing loss than non smokers [28]. The prevalence of smoking is on a high rise with rates significantly varying from $20 \%$ among UK population (Office for national Statistics, 2012) to $60 \%$ (World Health Organization, 2013). The audiological investigations in the present study revealed a significant difference was found between smokers and nonsmokers. Limited research is available on effects of smoking on cochlear function. Future research can target to study the effect of duration and amount of smoking on the hearing sensitivity.

\section{Summary}

The present study indicated that smoking is a risk factor that can lead to high frequency hearing loss. This study also revealed that chronic smokers could develop a risk of low frequency hearing loss. Moreover chronic smokers have reduced amplitude for OAEs especially in high frequencies as compared to nonsmokers. Hence, the results here suggest the need for OAEs \& HFA as important tools for early identification of hearing loss related to smoking.

\section{Future Directions}

In order to understand the effects of smoking on auditory system, future studies using evaluation procedures that may detect early hearing impairments such as HFA and OAE testing should be included. An insight into the blood viscosity levels, total nicotine content in the body of chronic smokers and their relation to hearing can be investigated in the future.

\section{References}

1. World Health Organization (2016) Tobacco. Fact sheet, WHO, Geneva, Switzerland.

2. Centres for Disease Control and Prevention (2012) The Health Consequences of Smoking: A report of the Surgeon General, USA.

3. Mc Ginnis J (1993) Actual cause of death in the United States, 2000. JAMA 270: 2207-2212.

4. Paschoal, Carolina Pamplona, Azevedo, Marisa Frasson de (2009) Cigarette smoking as a risk factor for auditory problems. Brazilian Journal of Otorhinolaryngology 75(6): 893-902.

5. Cocchiarella LA, Sharp DS, Persky VW (1995) Hearing threshold shifts, white-cell count and smoking status in working men. Occup Med 45(4): $179-185$

6. Stewart RD (1976) The effect of carbon monoxide on humans. J Occup Med 18: 304-309.

7. Chung DY, Wilson GN, Gannon RP, Mason K (1984) Factors affecting the prevalence of tinnitus. Audiology 23(5): 441-452.

8. Browning GG, Gatehouse S, Lowe GD (1986) Blood viscosity as a factor in sensory neural hearing impairment. Lancet 1(8473): 121-123.

9. McReynolds MC (2005) Noise induced hearing loss. Air Med J 24(2): 73-78.
10. Cruickshanks KJ, Tweed TS, Wiley TL, Klein BE, Klein R, et al. (2003) The 5-year incidence and the progression of hearing loss: the epidemiology of the hearing loss study. Arch Otolaryngol Head Neck Surg 129: 1041-1046.

11. David A Fabry, Evelyn P Davila, Kristopher L Arheart, Berrin Serdar, Noella A, et al. (2011) Secondhand Smoke Exposure and the Risk of Hearing Loss. Tob Control 20(1): 82-85.

12. Zeigelboim BS, Mangabeira Albernaz PL, Fukuda Y (2001) High frequency audiometry and chronic renal failure. Acta Otolaryngol 121(2): 245- 248.

13. Sahyeb DR, Costa Filho AO, Alvarenga KF (2003) Audiometria de alta frequência: estudo com indivíduos audiologicamente normais. Rev Bras Otorrinolaringol 69(1): 93-99.

14. Paschoal CP, de Azevedo MF ( 2009) Cigarette smoking as a risk factor for auditory problems. Braz J Otorhinolaryngol 75(6): 893-902.

15. Sharabi Y, Reshef Haran I, Burstein M, Eldad A (2002) Cigarette smoking and hearing loss: lessons from the young adult periodic examination in Israel (YAPEIS) database. Isr Med Assoc J 4(12): 1118-1120.

16. Nakanishi N, Okamoto M, Nakamura K, Suzuki K, Tatara K (2000) Cigarette smoking and risk for hearing impairment: A longitudinal study in Japanese male office workers. J Occup Environ Med 42(11): 1045-1049.

17. Negley C, Katbamna B, Crumpton C, Lawson GD (2007) Effects of cigarette smoking on distortion produce otoacoustic emissions. J Am Acad Audiol 18(8): 665-674.

18. World Health Organization (2017) Grades for hearing impairment. Prevention of blindness and deafness, WHO, Geneva, Switzerland.

19. De Oliveira DC, de Lima MA (2009) Low and high frequency tonal threshold audiometry: comparing hearing thresholds between smokers and non-smokers. Brazilian Journal of Otorhinolaryngology 75(5): 738-744.

20. Mizoue T, Miyamoto T, Shimizu T (2003) Combined effects of smoking and occupational exposure to noise on hearing loss in steel factory workers. Occup Environ Med 60(1): 56-59.

21. Glogau $O$ (1923) Nicotine poisoning of inner ear, a preliminary report from animal experimentation and microscopic findings. Laryngoscope 33(4): 262-266.

22. Richard HG (1996) Effects of air pollution on upper aerodigestive tract. Otolaryngol Head Neck Surg 114(2): 201-204.

23. Kumar A, Gulati R, Singhal S, Hasan A, Khan A (2013) The effect of smoking on the hearing status: A hospital based study. J Clin Diagn Res 7(2): 201-214.

24. Gaur K, Kasliwal N, Gupta R (2012) Association of smoking or tobacco use with ear disease among men: a retrospective study. Tob Induc Dis 10(1): 4.

25. Ohgami N, Kondo T, Kato M (2011) Effects of light smoking on extrahigh-frequency auditory thresholds in young adults. Toxicol Ind Health. 27(2): 143-147.

26. Dallos P (1992) The active cochlea. J Neurosci 12(12): 4575-4785.

27. Amir Houshang Mehrparvar, Abolfazl Mollasadeghi, Seyed Hesam Hashemi, Mohammad Javad Zare Sakhvidi, Mehrdad Mostaghaci, et al. (2015) Simultaneous effects of noise exposure and smoking on OAEs. Noise Health 17(77): 233-236.

28. Piers Dawes, Karen J Cruickshanks, David R Moore, Mark Edmondson Jones, Abby MC Cormack, et al. (2014) Cigarette Smoking, Passive Smoking, Alcohol Consumption and Hearing Loss. J Assoc Res Otolaryngology 15(4): 663-674. 
This work is licensed under Creative Commons Attribution 4.0 License DOI: $10.19080 /$ GJ0.2017.07.555720
Your next submission with Juniper Publishers will reach you the below assets

- Quality Editorial service

- Swift Peer Review

- Reprints availability

- E-prints Service

- Manuscript Podcast for convenient understanding

- Global attainment for your research

- Manuscript accessibility in different formats ( Pdf, E-pub, Full Text, Audio)

- Unceasing customer service

Track the below URL for one-step submission https://juniperpublishers.com/online-submission.php 